CASE REPORT

\title{
Life threatening hyperphosphataemia after administration of sodium phosphate in preparation for colonoscopy
}

\author{
I Azzam, Y Kovalev, S Storch, N Elias
}

Postgrad Med J 2004;80:487-488. doi: 10.1 136/pgmj.2003.017244

An elderly woman developed severe hyperphosphataemia hypocalcaemia, and cardiac arrest after oral administration of sodium phosphate in preparation for colonoscopy. This is an unusual complication and is attributed to decreased phosphate excretion by the kidneys. At increased risk are patients with impaired renal function, age more than 65 years, and presenting with intestinal obstruction or decreased intestinal motility, increased intestinal permeability, liver cirrhosis, or congestive heart failure. Though there are no accepted guidelines for anticipation and prevention of this adverse effect, it may be desirable to check serum phosphate concentrations before choosing the method for colonic preparation and before giving the second oral dose of sodium phosphate in patients at risk. Hyperphosphataemia should be suspected if a patient develops hypotension or neuromuscular irritability after administration of sodium phosphate. Haemodialysis for direct removal of phosphate and intravenous calcium for treatment of symptomatic hypocalcaemia may be life saving.

\footnotetext{
A
} dequate preparation of the colon is essential for satisfactory visualisation of the colonic mucosa. Two agents are commonly used for bowel preparation: polyethylene glycol or sodium phosphate based agents. Polyethylene glycol is a non-digestible, non-absorbable, osmotically balanced solution that cleans the bowel by washout of ingested fluids. Being neutral and iso-osmotic, it yields no net absorption or excretion of water and ions. ${ }^{1}$ The main disadvantage of colonic cleansing with polyethylene glycol is the bad taste and the need to ingest large volumes of the substance. Dibasic sodium phosphate is a powerful osmotic laxative, requiring ingestion of small volumes of fluid and thus providing an attractive alternative for colonic cleansing. Most studies comparing polyethylene glycol and sodium phosphate preparations showed similar compliance with both preparations, without affecting the quality of bowel cleansing. ${ }^{1}$ Sodium phosphate preparations are thought to be safe, though they may induce gastrointestinal adverse effects as well as hyperphosphataemia. ${ }^{2-6}$ We describe a patient who developed hyperphosphataemia and subsequent cardiac arrest after administration of both oral and rectal sodium phosphate in preparation for colonoscopy.

\section{CASE REPORT}

A 79 year old woman was admitted to the medical ward for evaluation of watery diarrhoea, epigastric pain and fatigue, which occurred during the preceding two months. There was a 30 year history of diabetes mellitus complicated with peripheral neuropathy as well as truncal obesity, hypothyroidism, non-alcoholic steatohepatitis with cirrhosis, portopulmonary hypertension, and mild chronic renal failure. The patient was regularly taking glibenclamide $5 \mathrm{mg}$ three times day, amitriptyline $10 \mathrm{mg}$ once a day, thyroxine sodium $100 \mu \mathrm{g}$ once a day, furosemide $40 \mathrm{mg}$ twice a day, isosorbide 5 mononitrate $40 \mathrm{mg}$ twice a day, and amlodipine $10 \mathrm{mg}$ once a day.

Her blood pressure on admission was $123 / 71 \mathrm{~mm} \mathrm{Hg}$ and heart rate 108 beats/min; the indurated liver edge was palpated $3 \mathrm{~cm}$ below the costal margin. Physical examination was otherwise unremarkable. Routine laboratory tests showed normal blood cell counts. The plasma concentration of calcium was $2.1 \mathrm{mmol} / \mathrm{l}$ and phosphorus $0.87 \mathrm{mmol} / \mathrm{l}$, blood glucose $11.7 \mathrm{mmol} / \mathrm{l}$, blood urea nitrogen $5.35 \mathrm{mmol} / \mathrm{l}$, and creatinine $114.9 \mu \mathrm{mol} / \mathrm{l}$. Urinalysis and examinations of stool specimens for occult blood, ova of parasites, clostridium toxin, as well as cultures for common pathogens, were negative. The electrocardiogram, chest and abdominal radiographic examinations were unremarkable.

The patient was prepared to undergo colonoscopy by oral administration of Soffadex. Each $5 \mathrm{ml}$ of Soffadex contains $2.4 \mathrm{~g}$ monobasic sodium phosphate and $0.9 \mathrm{~g}$ dibasic sodium phosphate; $45 \mathrm{ml}$ were given at 16:00 pm and $45 \mathrm{ml}$ given at 20:00 pm. In addition, Fleet enema was administered twice. Each enema contains $16 \mathrm{~g}$ of monobasic sodium phosphate and $6 \mathrm{~g}$ of dibasic sodium phosphate.

Because of unsatisfactory cleaning, the same regimen was repeated on the subsequent day. The morning of the scheduled colonoscopy the patient became stuporous, the blood pressure was $62 / 40 \mathrm{~mm} \mathrm{Hg}$, the heart rate 108 beats/ min, and respirations were $40 / \mathrm{min}$. The arterial oxygen saturation was $75 \%$. Blood tests showed severe hyperphosphataemia $(7.75 \mathrm{mmol} / \mathrm{l})$, hypocalcaemia (total calcium $1.35 \mathrm{mmol} / \mathrm{l}$ ), metabolic acidosis ( $\mathrm{pH} 7.0$ and plasma bicarbonate $9 \mathrm{mg} / 100 \mathrm{ml}$ ), exacerbation of renal failure (creatinine $176.8 \mu \mathrm{mol} / \mathrm{l}$, blood urea nitrogen $10.3 \mathrm{mmol} / \mathrm{l}$ ), and she became oliguric. The patient was mechanically ventilated. Intravenous saline, dopamine, and bicarbonate were administered. At this time asystole was noticed on the electrocardiographic monitor.

After successful resuscitation, the patient underwent haemodialysis. After first dialysis, the serum phosphorus concentration decreased to $3.8 \mathrm{mmol} / \mathrm{l}$, calcium was $1.1 \mathrm{mmol} / \mathrm{l}$, blood urea nitrogen $6.42 \mathrm{mmol} / \mathrm{l}$, and creatinine $141.4 \mu \mathrm{mol} / \mathrm{l}$. Haemodialysis was repeated daily until normalisation of the serum phosphorus concentrations two days later. Calcium gluconate was administered intravenously as necessary to keep the calcium levels in the low normal range.

Within five days, the patient's general state and results of laboratory examinations returned to baseline and she was discharged from hospital. A barium enema was performed subsequently showing normal colonic mucosa.

\section{DISCUSSION}

Severe hyperphosphataemia and cardiac arrest occurred in this patient after the administration of sodium phosphate in preparation for colonoscopy. Mild, asymptomatic hyperphosphataemia up to 2-3 times above normal phosphorus levels 
occurs in nearly $25 \%$ of individuals with normal renal function after administration of sodium phosphate-containing colonic preparations. ${ }^{2}$ Severe, symptomatic hyperphosphataemia after the administration of sodium phosphate was sporadically reported. This occurs mainly in patients with impaired renal function and is attributed to decreased phosphate excretion by the kidneys. ${ }^{3}$ Age related decline in renal function may be present. This is often overlooked, in spite of $50 \%$ or greater decline in glomerular filtration rate, when the serum urea and creatinine values are in the normal range. ${ }^{4}$ There is no accepted cut off level for serum creatinine above which sodium phosphate colonic preparation is contraindicated. The same is true of creatinine clearance. Other circumstances linked with increased risk to develop hyperphosphataemia are: Hirschsprung's disease, faecal impaction, or functional intestinal obstruction where increased gastrointestinal phosphate absorption may occur, elderly age because of the diminished intestinal motility, ${ }^{6}$ and increased intestinal permeability in the presence of inflammatory intestinal disorders. ${ }^{7}$ Several factors may have contributed to hyperphosphataemia in our patient: the higher than usual phosphate dose because of unsatisfactory bowel cleansing, presence of mild chronic renal failure, and probably sluggish intestinal peristalsis caused by immobility.

The signs and symptoms of acute hyperphosphataemia are mainly the consequence of concomitant hypocalcaemia, which results from formation of insoluble calcium phosphate precipitates. These include neuromuscular irritability, tetany, hypotension, and increased QT interval in the electrocardiogram. Calcium phosphate deposition may lead to vascular, ocular, periarticular, and cardiac calcifications. Acute renal failure may develop because of direct phosphorus toxicity ${ }^{8}$ or to calcium phosphate deposition in the renal interstitium. ${ }^{9}$ There is controversy whether to treat such hypocalcaemia with calcium infusion. ${ }^{10}$ Such treatment carries the risk of calcium phosphate precipitation in vital organs. It is empirically recommended to bring calcium levels to the low-normal range. Attempts to restore normal levels of calcium should be withheld until the plasma phosphorus

\section{Learning points}

Precautions to prevent severe hyperphosphataemia following preparation for colonoscopy

1. Consider possible risk factors

- Renal failure.

- Age 65 years or older.

- Intestinal obstruction.

- Decreased intestinal motility.

- Increased intestinal permeability.

- Systemic disease: congestive heart failure, liver cirrhosis.

\section{Prevention: double checking serum phosphate} levels

- Before choosing the agent for colonic preparation.

- Before giving the second oral dose in patients at risk.

3. Awareness of symptoms of phosphate intoxication

- Hypotension, neuromuscular irritability, tetany. level returns to normal. Because our patient was oliguric while having extreme hyperphosphataemia, we performed repeated haemodialysis. The serum phosphorus decreased to $3.8 \mathrm{mmol} / \mathrm{l}$ after the first session of haemodialysis and to normal levels after three consecutive days of intermittent haemodialysis.

There are no accepted guidelines for anticipation and prevention of severe hypophosphataemia after administration of sodium phosphate in preparation for colonoscopy. The manufacturer contraindicates the use of Soffadex (Dexxon) in patients presenting with congenital megacolon or having bowel obstruction. At increased risk of developing hyperphosphataemia are patients with one or several of the following: age more than 65 years, impaired renal function, intestinal obstruction, decreased intestinal motility, increased intestinal permeability, liver cirrhosis, and congestive heart failure. It may be desirable to test serum phosphate levels before choosing the method for colonic preparation and to repeat the measurement before giving the second oral dose of sodium phosphate in patients at increased risk. In patients considered to be at increased risk of phosphate intoxication, preparation of the bowel with polyethylene glycol may be preferable. Hyperphosphataemia should be suspected if a patient develops hypotension or neuromuscular irritability after administration of sodium phosphate. Haemodialysis for direct removal of phosphate and intravenous calcium for treatment of symptomatic hypocalcaemia may be life saving.

\section{Authors' affiliations}

I Azzam, Y Kovalev, N Elias, Department of Internal Medicine A, Bnai Zion Medical Center and Bruce Rappaport Faculty of Medicine, Technion-Israel Institute of Technology, Haifa, Israel

S Storch, Department of Nephrology, Bnai Zion Medical Center and Bruce Rappaport Faculty of Medicine, Technion-Israel Institute of Technology, Haifa, Israel

Correspondence to: Dr I Azzam, Department of Internal Medicine A, Bnai Zion Medical Center, Haifa 31048, PO Box 4940, Israel; Azzami76@yahoo.com

Submitted 17 November 2003

Accepted 20 February 2004

\section{REFERENCES}

1 Hsu CW, Imperiale TF. Meta-analysis and cost comparison of polyethylene glycol lavage versus sodium phosphate for colonoscopy preparation. Gastrointest Endosc 1998;48:276-82.

2 Lieberman D, Ghormley J, Flora K. Effects of oral sodium phosphate colon preparation on serum electrolytes in patients with normal serum creatinine. Gastrointest Endosc 1995;43:467-9.

3 Fine A, Patterson J. Severe hyperphosphatemia following phosphate administration for bowel preparation in patients with renal failure: two cases and a review of the literature. Am J Kidney Dis 1997:29:103-5.

4 Beloosesky Y, Grinblat J, Weiss A, et al. Electrolyte disorders following oral sodium phosphate administration for bowel cleansing in elderly patients. Arch Intern Med 2003;163:803-8

5 Fass R, Do S, Hixson U. Fatal hyperphosphatemia following fleet phosphorsoda in a patient with colonic ileus. Am J Gastroenterol 1993:88:929-32.

6 Vukasin P, Weston LA, Beart RW. Oral Fleet phosphor-soda laxative-induced hyperphosphatemia and hypocalcemia tetany in an adult: report of a case. Dis Colon Rectum 1997;40:497-9.

7 Pantzar N, Ekstrom GM, Wang Q. Mechanism of increased intestinal $\left\{{ }^{51} \mathrm{Cr}\right\}$ EDTA absorbtion during experimental colitis in the rat. Dig Dis Sci 1994;39:2327-33.

8 Zager R. Hyperphosphatemia: a factor that provokes severe experimental acute renal failure. J Lab Clin Med 1982;100:230-9.

9 Herbert LA, Lemann J, Peterson JR, et al. Studies of the mechanism by wich phosphate infusion lowers serum calcium concentration. J Clin Invest 1966;45: 1886-94.

10 Sutters M, Gaboury CL, Bennett WM. Severe hyperphosphatemia and hypocalcemia: a dilemma in patient management. J Am Soc Nephrol 1996;7:2056-61 